\title{
Hybrid Fuzzy Controller Based Frequency Regulation in Restructured Power System
}

\author{
P. Anitha1, P. Subburaj² \\ ${ }^{1}$ Department of Electrical Engineering, Anna University, Thoothukudi, India \\ ${ }^{2}$ Department of Electrical Engineering, National Engineering College, Kovilpatti, India \\ Email: anithap18@gmail.com
}

Received 5 April 2016; accepted 8 May 2016; published 13 May 2016

Copyright (C) 2016 by authors and Scientific Research Publishing Inc.

This work is licensed under the Creative Commons Attribution International License (CC BY).

http://creativecommons.org/licenses/by/4.0/

c) (i) Open Access

\begin{abstract}
This paper discusses the implementation of Load Frequency Control (LFC) in restructured power system using Hybrid Fuzzy controller. The formulation of LFC in open energy market is much more challenging; hence it needs an intelligent controller to adapt the changes imposed by the dynamics of restructured bilateral contracts. Fuzzy Logic Control deals well with uncertainty and indistinctness while Particle Swarm Optimization (PSO) is a well-known optimization tool. Abovementioned techniques are combined and called as Hybrid Fuzzy to improve the dynamic performance of the system. Frequency control of restructured system has been achieved by automatic Membership Function (MF) tuned fuzzy logic controller. The parameters defining membership function has been tuned and updated from time to time using Particle Swarm Optimization (PSO). The robustness of the proposed hybrid fuzzy controller has been compared with conventional fuzzy logic controller using performance measures like overshoot and settling time following a step load perturbation. The motivation for using membership function tuning using PSO is to show the behavior of the controller for a wide range of system parameters and load changes. Error based analysis with parametric uncertainties and load changes is tested on a two-area restructured power system.
\end{abstract}

\section{Keywords}

Fuzzy Logic Controller, Membership Function, Particle Swarm Optimization, Load Frequency Control, Bilateral Market

\section{Introduction}

Frequency regulation service in energy market is one of the profitable ancillary services. Its main objectives are to keep the tie-line power exchanges within the scheduled limits and to maintain zero change in frequency. Since 
frequency control in a complex and competitive environment is a tedious process, the control technique must be more intelligent and adaptive for a changing environment. More research work has been carried on Frequency control in restructured power system [1]-[7]. Possible issues in Frequency control in energy market were discussed in [1]. Vertically integrated structure and bilateral based scheme modeling were discussed in [2] [3]. Different approaches like pluralistic and hierarchical methods were discussed and quoted in [4]. A decentralized approach based on control theory was formulated and analyzed in [5]. Some researchers have proposed a frequency control logic based on fuzzy logic controller [6], reinforcement learning [7] and artificial neural network [8] in interconnected power systems.

The Load Frequency Control (LFC) issue as an ancillary service represents an important role to maintain an acceptable level of efficiency, quality, and reliability in a deregulated power system environment. Many researchers have started to analyze possible new LFC schemes and regulation solutions, with paradigms suited for the energy market scenarios [4]-[9]. In this paper bilateral contract scheme under restructured power system is taken for analysis. The objective of the proposed controller in bilateral market is to reduce damping of frequency and tie-line power deviations oscillation for all admissible uncertainties and load changes. So, three different testing conditions are taken to test the robustness of the controllers.

This paper is organized as follows: Generalized bilateral scheme is presented in section 2 and Generation Participation Matrix is defined in this section. System taken for investigation is defined in section 3. Fuzzy rule based control scheme for a bilateral based restructured power system is presented in section 4 . Automatic membership function tuning using PSO based controller is discussed in section 5. Simulation results for different cases are presented in Section 6. The objective of this work is to formulate the dynamic LFC and evaluate and analyze the LFC based on different performance measures under parametric uncertainties.

\section{Generalized Bilateral Scheme}

Energy market scenarios are adapting changes every day to achieve the goal of better performance and efficiency in restructured power systems. In this paper, bilateral based market is taken for analysis. A general configuration for the LFC [2] [3] in a deregulated environment is shown in Figure 1.

Bilateral based deregulated environment consist of an Independent System Operator (ISO), Distribution Companies (DISCOs), Generation Companies (GENCOs), and Transmission Companies (TRANSCOs). In the new environment, DISCOs may contract power from any GENCOs and ISO have to supervise these contracts

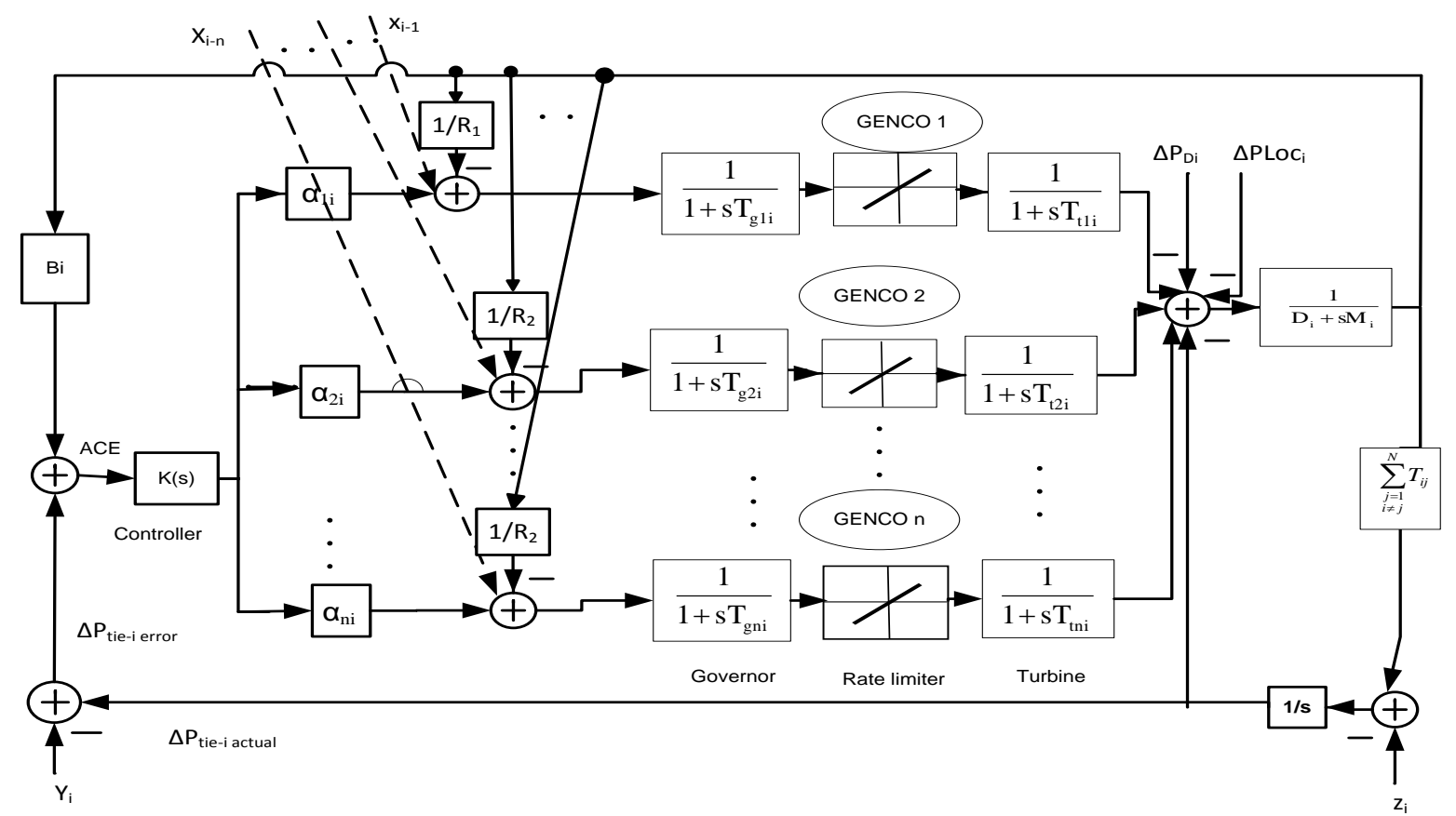

Figure 1. Bilateral based LFC model. 
[9]. The "Generation Participation Matrix (GPM)" shows the participation factor of each Genco in the considered control areas, and each control area is determined by a Disco. The rows of a GPM correspond to Gencos and the columns to the control areas that contract power. For example, the GPM for a large scale power system with $m$ control areas (Discos) and $n$ Gencos, has the following structure, in which gpf $f_{i j}$ refers to "generation participation factor" and shows the participation factor of Genco $i$ in total load following the requirement of DISCO $j$ based on a specified bilateral contract [10] in Equation (1).

New information signals due to possible various contracts between Disco $i$ and other Discos and Gencos are shown as dashed arrows in Figure 1.

$$
\begin{aligned}
G P M & =\left[\begin{array}{ccccc}
g p f_{11} & g p f_{12} & \cdots & g p f_{1(m-1)} & g p f_{1 m} \\
g p f_{21} & g p f_{22} & \cdots & g p f_{2(m-1)} & g p f_{2 m} \\
\vdots & \vdots & & \vdots & \vdots \\
g p f_{(n-1) 1} & g p f_{(n-1) 2} & \cdots & g p f_{(n-1)(m-1)} & g p f_{(n-1) m} \\
g p f_{n 1} & g p f_{n 2} & \cdots & g p f_{n(m-1)} & g p f_{n m}
\end{array}\right] \\
\Delta P_{\text {tie-i,error }} & =\Delta P_{\text {tie-i,actual }}-\sum(\text { Total export power - Total import Power }) \\
& =\Delta P_{\text {tie-i,actual }}-y_{i}
\end{aligned}
$$

where

$$
\begin{gathered}
y_{i}=\sum_{\substack{j=1 \\
j \neq i}}^{N}\left(\sum_{k=1}^{n} g p f_{k j}\right) \Delta P_{L j}-\sum_{k=1}^{n}\left(\sum_{\substack{j=1 \\
j \neq i}}^{N} g P f_{k j}\right) \Delta P_{L i} \\
\sum_{i=1}^{n} g p f_{i j}=1 \\
\sum_{k=1}^{n} \alpha_{k i}=1 ; 0 \leq \alpha_{k i} \leq 1 \\
\Delta P_{m i}=\sum_{j=1}^{N} g p f_{i j} \Delta P_{L j} \\
\Delta P_{\text {tie-i, scheduled }}=\sum_{\substack{j=1 \\
j \neq i}}^{N}\left(\sum_{k=1}^{n} g p f_{k j}\right) \Delta P_{L j}-\sum_{k=1}^{n}\left(\sum_{\substack{j=1 \\
j \neq i}}^{N} g p f_{k j}\right) \Delta P_{L i}
\end{gathered}
$$

where $\Delta P_{d i}$ (Figure 1) is the area load disturbance, $\Delta P_{L o c-i}$ is the contracted load demand (contracted and uncontracted) in area $i$, and $\Delta P_{\text {tie--,actual }}$ is the actual tie-line power in area $i$. Using Equation (2), the scheduled tie-line power $\left(\Delta P_{\text {tie-I,scheduled }}\right)$ can be calculated using Equation (7).

In the bilateral LFC structure, Control is highly decentralized. Each Load Matching Contract requires a separate control process, yet these control processes must cooperatively interact to maintain system frequency and minimize the area control error [4], where

$X_{i}$ Generator participation factor under contract.

$Y_{i}$ Difference of import and export power.

$Z_{i}$ Tie-line coefficient.

$\Delta f_{i}$ frequency deviation.

$B_{i}$ frequency bias.

$\Delta P_{\text {tie } \_}$net tie-line power flow.

$\Delta P_{d i}$ area load disturbance between areas $i$ and $j$.

$\alpha$ area control error (ACE) participation factor.

$\Delta P_{L i}$ contracted demand of area $i$.

$\Delta P_{\text {mi }}$ power generation of Genco $i$.

$\Delta P_{\text {Loc } \_i}$ total local demand (contracted and uncontracted) in area $i$.

$\Delta P_{\text {tie_i, actual }}$ actual $\Delta P_{\text {tie_- } i \text {. }}$ 


\section{System Modeling}

Consider the load frequency control problem for a two-area power system as shown in Figure 2. Each control area has the structure as shown in Figure 1. The control area considered here is simplified power systems consisting of two generating units of thermal reheat system [11]. According to [12], the collective performance of all generators in the system is the interesting part in the analysis of LFC. Genco's considered here are reheat thermal units. The inter-machine oscillations are not considered. It is assumed that the response of all generators to changes in system load are coherent and can be represented by an equivalent generator, which has an inertia constant and a damping constant equal to the sum of the inertia constants and damping constants of all the generating units.

The model is simple but captures the essential dynamics of a power system and has been widely used for LFC design purpose. The load frequency control problem for a multi-area power system requires that not only the frequency deviation of each area must return to its nominal value but also the tie-line power flows must return to their scheduled values. So a composite variable, the area control error (ACE), is used as the feedback variable to ensure the two objectives.

For Area \#i, the area control error is defined as

$$
A C E_{i}=\Delta P_{\text {tie-i }}+B_{i} \Delta f_{i}
$$

and the feedback control for Area \#i take the form

$$
C_{i}=-k_{p i}(s) A C E_{i}-k_{I i}(s) \int A C E_{i}
$$

The transfer functions of the governor, the turbine, and the rotor inertia and load for Area $\# i$ are denoted by $G_{g i}(s), G_{t i}(s)$, and $G_{p i}(s)$, respectively. The transfer functions of the above mentioned are represented as

$$
\begin{gathered}
G_{g i}(s)=\frac{1}{1+s T_{g i}} \\
G_{t i}(s)=\frac{1}{1+s T_{t i}} \\
G_{p i}(s)=\frac{K_{p i}}{1+s T_{p i}}
\end{gathered}
$$

then the transfer function from $u_{i}$ to $\Delta f_{i}$ can be easily found as

$$
G_{i}(s)=\frac{G_{p i} G_{t i} G_{g i}}{1+G_{p i} G_{t i} G_{g i} / R_{i}}
$$

The transfer function for Area \#i.

$$
P_{i}(s)=G_{i}(s) B_{i}
$$

The system parameters taken for investigation [13] are given in Appendix A.

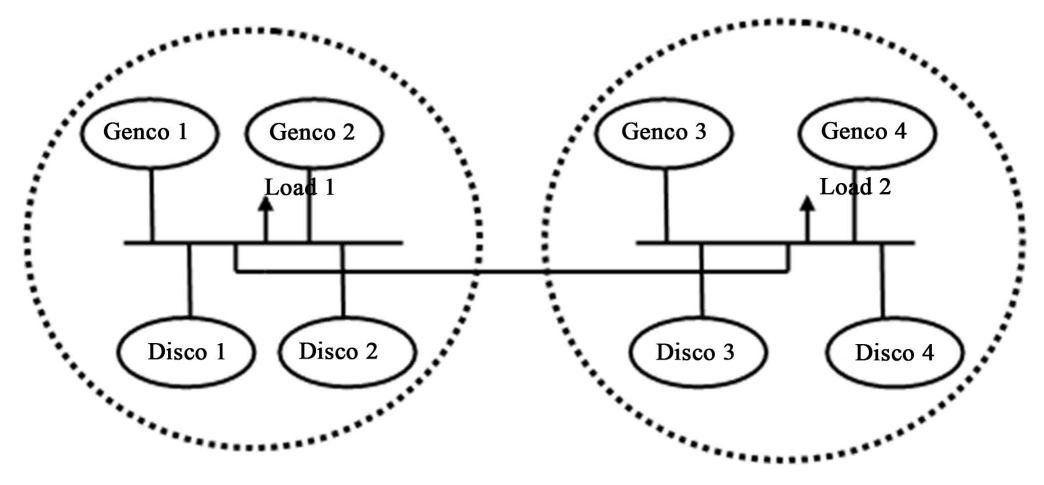

Figure 2. Two control area. 


\section{Fuzzy Rule Based Controller Design}

Due to the increasing complexity and changing structure of power systems, it needs an intelligent controller as an alternative to the conventional control methodology. Fuzzy Logic Control would serve the better role in load frequency control in restructured power system. Fuzzy controller consists of three main stages, namely the Fuzzification interface, the Inference rules engine and Defuzzification interface.

For LFC, ACE and its derivative are chosen as inputs and Proportional and Integral Gains are taken as control outputs of the fuzzy controller. Gaussian Membership Functions (MFs) is chosen for obtaining fast response from the system. The MFs were named NL (Negative Large), NM (Negative Medium), NS (Negative Small), Z (Zero), PS (Positive Small), PM (Positive Medium) and PL (Positive Large) followed by error(E) and Derivative error (DE). In [14], Ziegler-Nichols proposed a heuristic method to quickly adjust the controller parameters P, PI, PID. The MFs ranges of the inputs (Ace \& dAce) and control outputs $\left(K_{P} \& K_{i}\right)$ for area $i$ are determined based on the experiments with classical (Ziegler-Nichols Tuned) PI controller. These two input signals are used as rule-antecedent in the formation of rule base, and the control outputs are used to represent the contents of the rule-consequent in performing the rule base [7].

Each fuzzy rule is in the form,

$$
\begin{aligned}
& \text { If } A C E_{i}(t) \text { is } M F_{n 1} \& \triangle A C E_{i}(t) \text { is } M F_{n 2} \\
& \text { Then } K_{P} \text { is } y_{n} \& K_{I} \text { is } z_{n} .
\end{aligned}
$$

where $M F_{n j}$ is a membership function and $y_{n}$ and $z_{n}$ are singleton values.

$$
M F_{n j}=\exp \left\{-\frac{\left(x-C_{n j}\right)^{2}}{\sigma_{n j}^{2}}\right\}
$$

where $C_{n j}$ is mean and $\sigma_{n j}$ is standard deviation of the membership function.

AND operation is used and is given by

$$
\mu_{n}=\prod_{j=1}^{2} M F_{n j}
$$

The output $K_{P}$ and $K_{I}$ calculated by

$$
\begin{gathered}
K_{P}=\sum_{n=1}^{k} \frac{y_{n} \mu_{n}}{\mu_{n}} \\
K_{I}=\sum_{n=1}^{k} \frac{z_{n} \mu_{n}}{\mu_{n}}
\end{gathered}
$$

" $k$ " refers total number of rules.

Based on the knowledge gathered from classical (Z-N) controller, fuzzy rules are formed and the appropriate rules for $K_{i}$ and $K_{p}$ are given in Table 1 .

Table 1. Rule base for conventional fuzzy controller.

\begin{tabular}{cccccccc}
\hline & NLE & NME & NSE & ZE & PSE & PME & PLE \\
\hline NLDE & PL & PL & PL & PM & PM & PS & Z \\
NMDE & PL & PM & PM & PM & PS & Z & NS \\
NSDE & PL & PM & PS & PS & Z & NS & NM \\
ZDE & PM & PM & PS & Z & NS & NM & NM \\
PSDE & PM & PS & Z & NS & NS & NM & NL \\
PMDE & PS & Z & NS & NM & NM & NM & NL \\
PLDE & Z & NS & NM & NM & NL & NL & NL \\
\hline
\end{tabular}




\section{Hybrid Fuzzy-Automatic MF Tuning Using PSO Controller}

PSO is a novel population based meta heuristic, which utilize the swarm intelligence generated by the cooperation and competition between the particles in a swarm and has emerged as a useful tool for engineering optimization. It has also been found to be robust in solving problems featuring nonlinearity, non-differentiability and high dimensionality.

In PSO, each particle is flown through the multidimensional search space, adjusting its position in search space according to its own experience or knowledge and that of neighboring particles. Therefore, a particle makes use of the best position encountered by itself and the best position of its neighbors to position itself toward an optimum solution. The effect is that particles fly toward the global minimum, while still searching a wide area around the best solution. The performance of each particle is measured according to a predefined fitness function which is related to the problem being solved [15]. The upper limit of the random value is a system parameter specified by the user. The larger the upper limit, the more the trajectory of the particles oscillates. Smaller values ensure smooth trajectories. This upper limit prevents particles from moving too rapidly from one region in search space to another. Vmax is usually initialized as a function of the range of the problem. The inertia weight controls the influence of previous velocities on the new velocity. Large inertia weights cause larger exploration of the search space, while smaller inertia weights focus the search on a smaller region [16]. In this proposed PSO, the inertia weight $(w)$ is started with a large value, which is decreased over time.

PSO starts with a population of random solutions "particles" in a D-dimension space. The ith particle is represented by $X_{i}=\left(x_{i 1}, x_{i 2}, \cdots, x_{i D}\right)$. Each particle keeps track of its coordinates in hyperspace, which are associated with the fittest solution it has achieved so far. The value of the fitness for particle $\mathrm{i}$ (pbest) is also stored as $P_{i}=\left(p_{i 1}, p_{i 2}, \cdots, p_{i D}\right)$. The global version of PSO keeps track of the overall best value (gbest), and its location, obtained thus far by any particle in the population. PSO consists of, at each step, changing the velocity of each particle toward its pbest and gbest according to Equation (15). The velocity of particle $\mathrm{i}$ is represented as $V_{i}=\left(v_{i 1}, v_{i 2}, \cdots, v_{i D}\right)$. Acceleration is weighed by a random term, with separate random numbers being generated for acceleration toward $\mathrm{p}$ best and $\mathrm{g}$ best. The position of the ith particle is then updated according to Equation (16) [17]. Figure 3 shows the flowchart of the proposed PSO algorithm.

$$
\begin{gathered}
v_{i D}=w \times v_{i D}+c 1 \times \operatorname{rand}() \times\left(p_{i d}-x_{i d}\right)+c 2 \times \operatorname{rand}() \times\left(p_{g d}-x_{i d}\right) \\
x_{i d}=x_{i d}+v_{i d}
\end{gathered}
$$

It should be noted that choice properly fitness function is very important in synthesis procedure. Because different fitness functions promote different PSO behaviors, which generate fitness value providing a performance measure of the problem considered [1]. Integral of Squared Error (ISE), criterion integrates the square of the error over time. ISE will penalize large errors more than smaller ones (since the square of a large error will be much bigger). ISE is used as fitness function in the proposed PSO.

$$
f(I S E)=\int_{0}^{T}\left(\Delta P_{\text {tie- } i}+B_{i} \Delta f\right)^{2} \mathrm{~d} T
$$

\section{Simulation Results}

In order to illustrate the behavior of the proposed control strategy some simulations has been carried out. The system parameter of the test system is given in Appendix A.

Consider a system where all GENCOs in each area participate in LFC, i.e. ACE participation factors are $\alpha_{1}=0.75, \alpha_{2}=1-\alpha_{1}=0.25 ; \alpha_{3}=0.5, \alpha_{4}=1-\alpha_{3}=0.5$

$$
G P M=\left|\begin{array}{cccc}
0.5 & 0.25 & 0 & 0.3 \\
0.2 & 0.25 & 0 & 0 \\
0 & 0.25 & 1 & 0.7 \\
0.3 & 0.25 & 0 & 0
\end{array}\right|
$$

Case I: In this case it is assumed that all Gencos are participating in the LFC task as per contract. It is assumed that a large step load 0.05 p.u MW is demanded by each DISCOs in all areas. Frequency response for 


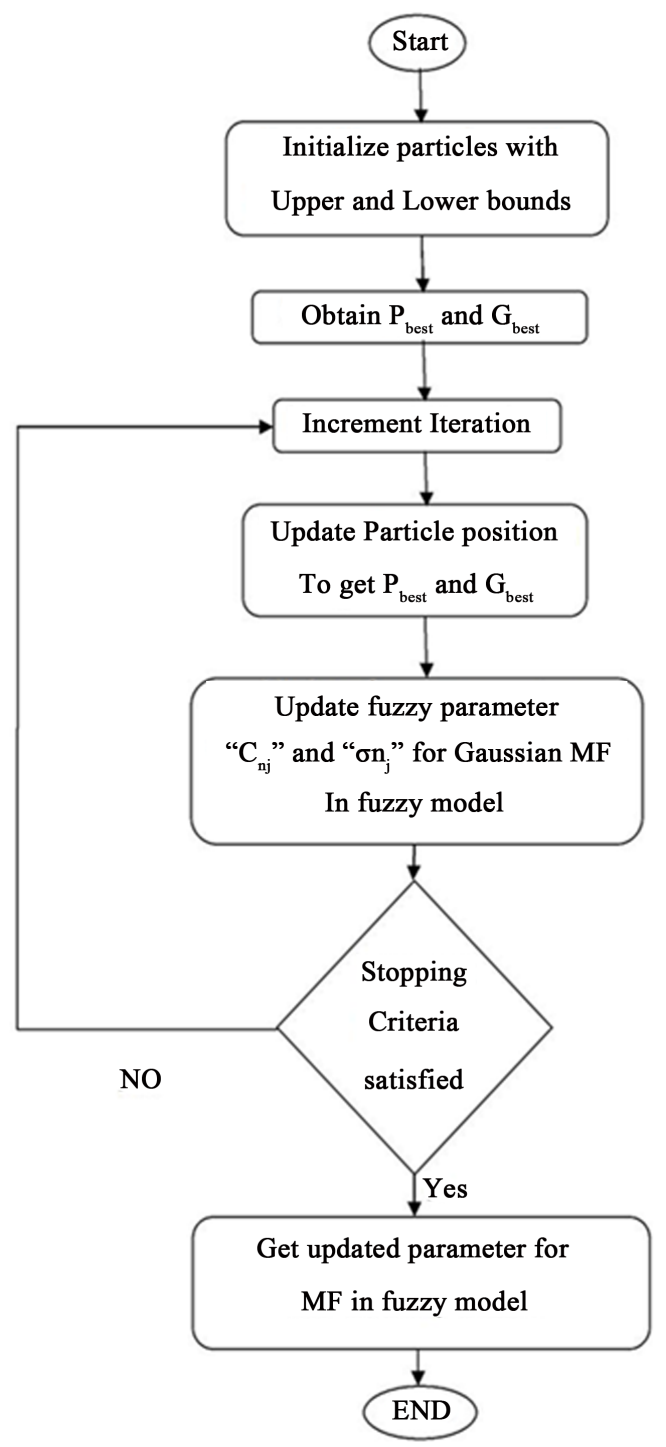

Figure 3. Flow chart-MF tuning using PSO.

both areas, tie line power exchange are shown in Figures 4-6. The actual power generated by each Gencos reach the desired value is given in Table 2. The membership function parameters Mean $\left(C_{n j}\right)$ and standard Deviation $\left(\sigma_{n j}\right)$ before and after updation are given in Table 3.

Case II: It is assumed that the rotating mass and load pattern parameters $D_{i}$ and $M_{i}$ have uncertain values in each control area. In addition to case I, 25\% decrease in parametric uncertainties is taken. The results are shown in Figures 7-9. Simulation results show that all the Hybrid Fuzzy controllers track the load fluctuations possibly good. Their overshoot \& settling time is better than conventional Fuzzy controllers.

Case III: Consider Case II, in addition to the specified contracted load demand and 25\% decrease in $D_{i}$ and $M_{i}$, a bounded random step load change $\left(\Delta P_{d i}\right)$ as an uncontracted demand appears in each control area.

$$
(-0.05 \mathrm{pu}) \leq \Delta P_{d i} \leq(+0.05 \mathrm{pu})
$$

The main aim of this test is to check the robustness of the proposed controller against uncertainties and random load disturbance. The load pattern applied to areas is shown in Figure 10. The results are shown in Figure 11 and Figure 12. The performance measures like overshoot and settling time are compared in Table 4.

The optimum values of the Proportional and Integral Gains of the different controllers for the aforementioned cases are shown in Table 5. 


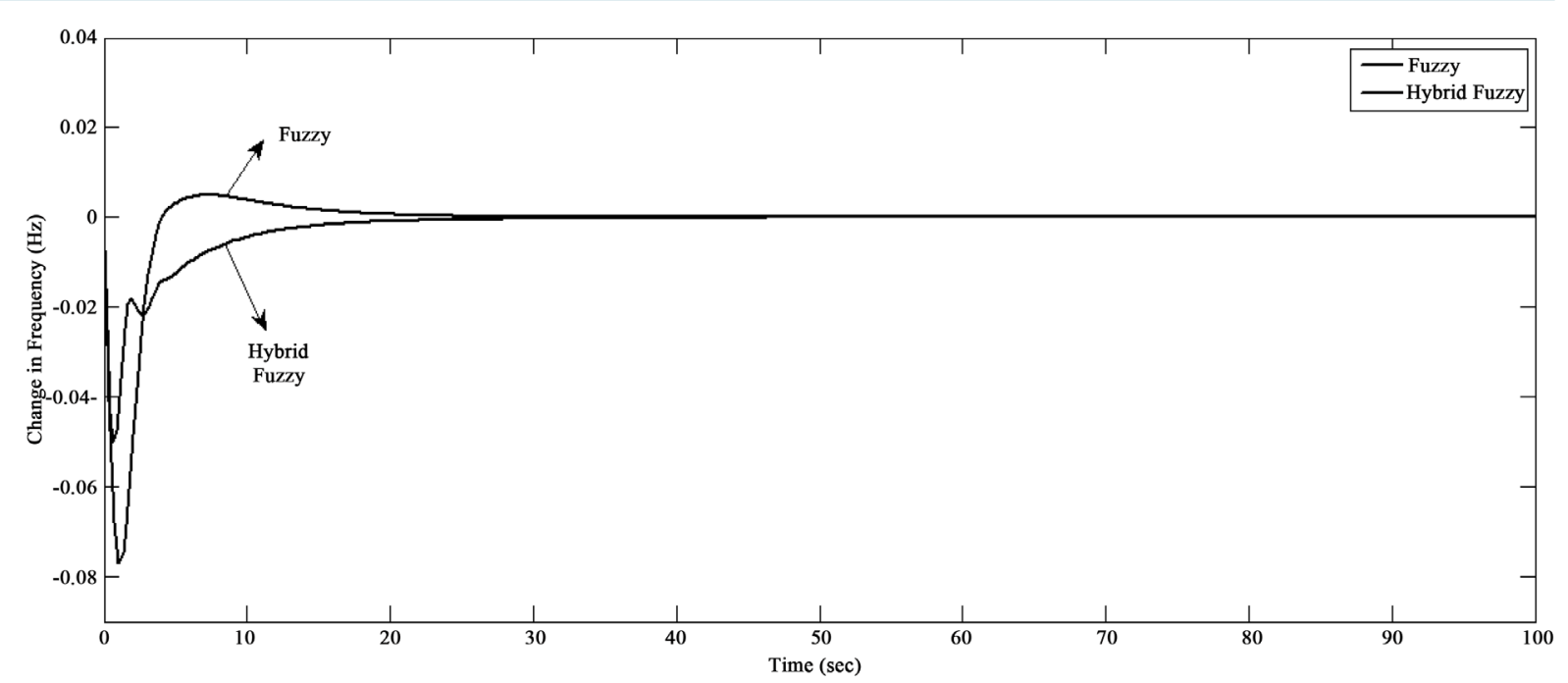

Figure 4. Frequency deviation for Area 1 (Case I).

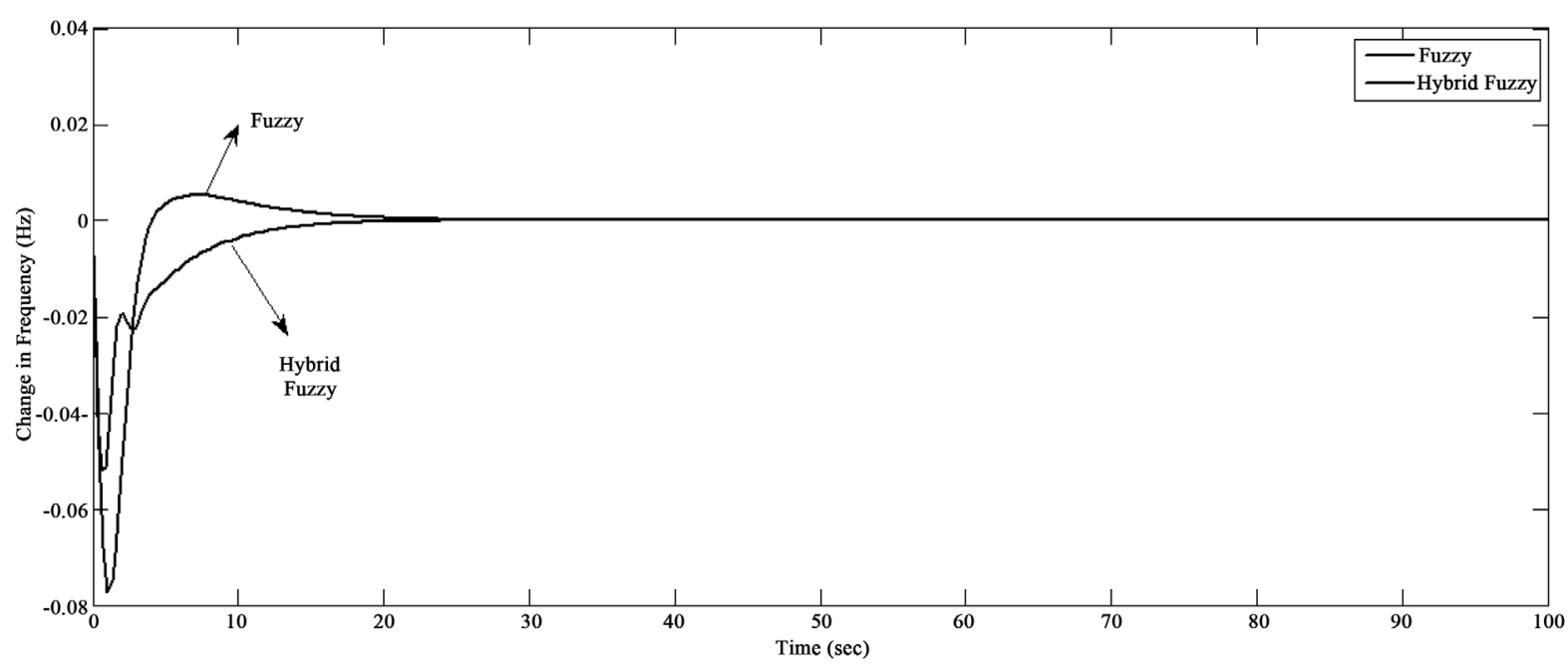

Figure 5. Frequency deviation for Area 2 (Case I).

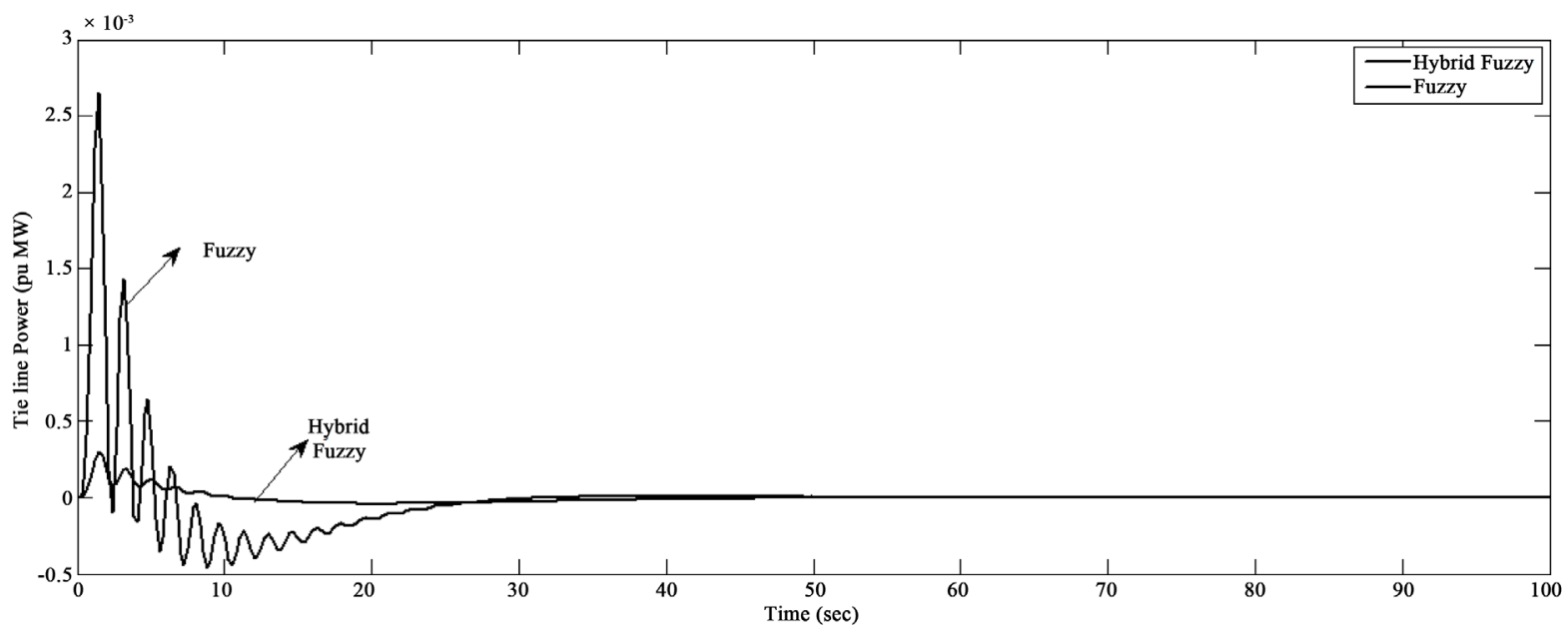

Figure 6. Tie-line power (Case I). 


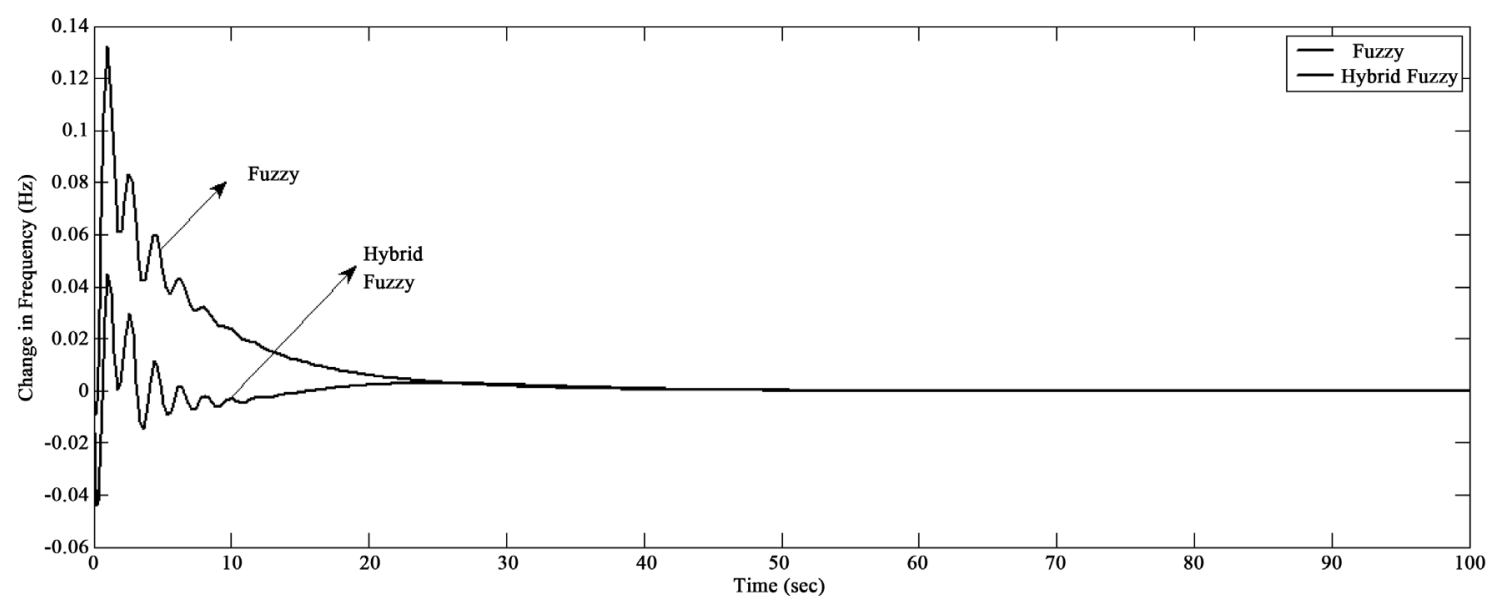

Figure 7. Frequency deviation for Area 1 (Case II).

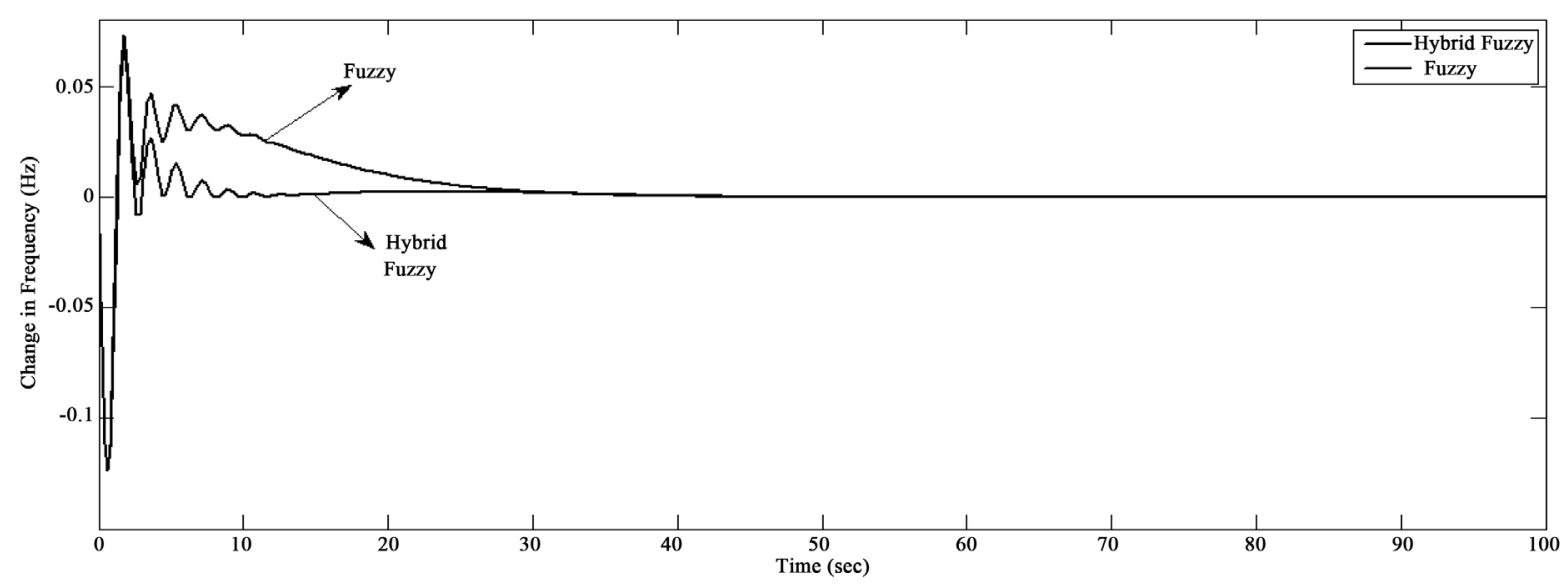

Figure 8. Frequency deviation for Area 2 (Case II).

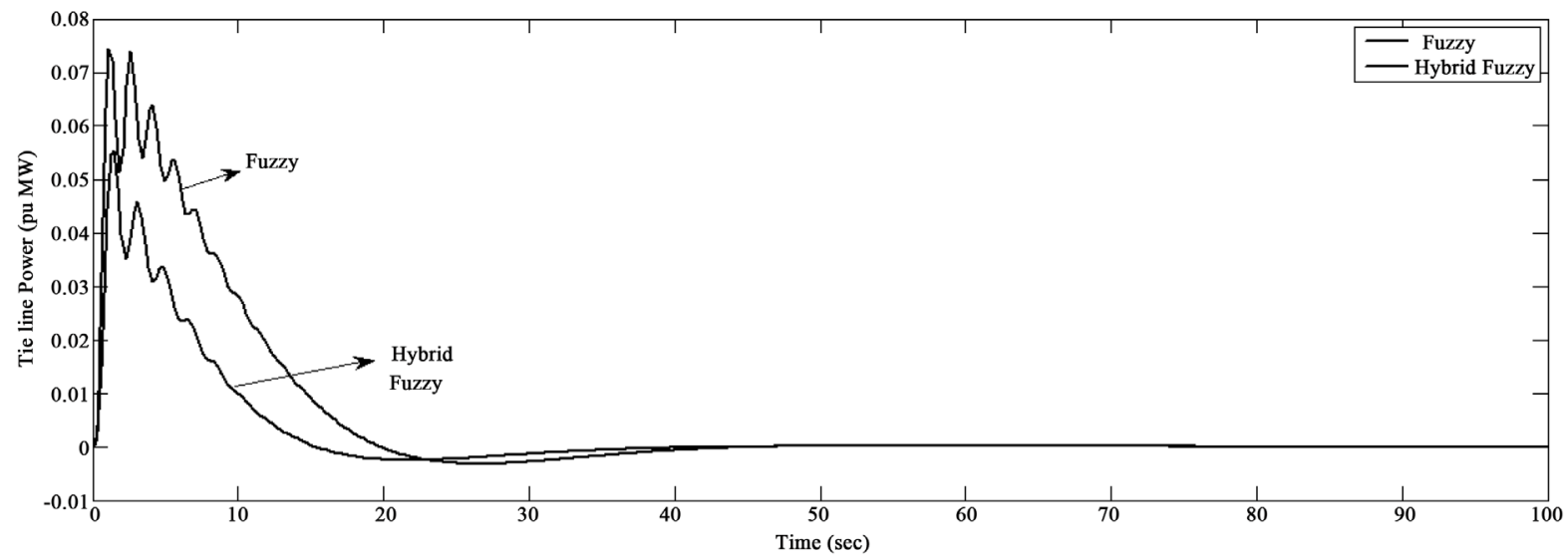

Figure 9. Tie-line power (Case II).

Table 2. Generated power in response to Case 1.

\begin{tabular}{ccccc}
\hline Genco & 1 & 2 & 3 & 4 \\
\hline $\mathrm{P}_{\mathrm{mi}}$ (puMW) & 0.0525 & 0.0225 & 0.0975 & 0.0275 \\
\hline
\end{tabular}




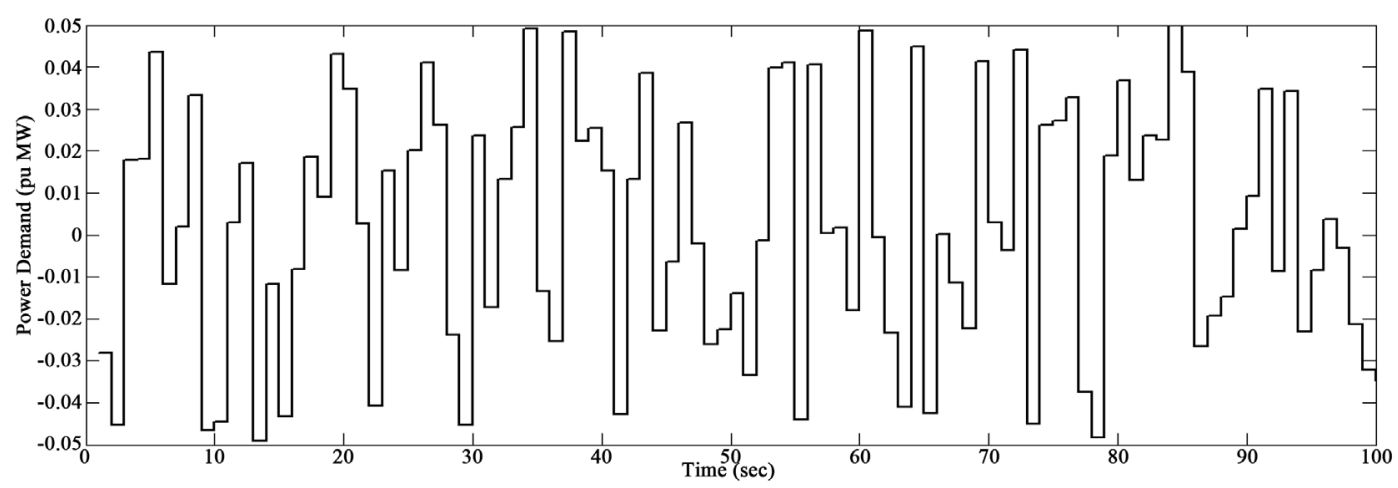

Figure 10. Random load pattern applied to Area 1 \& Area 2.

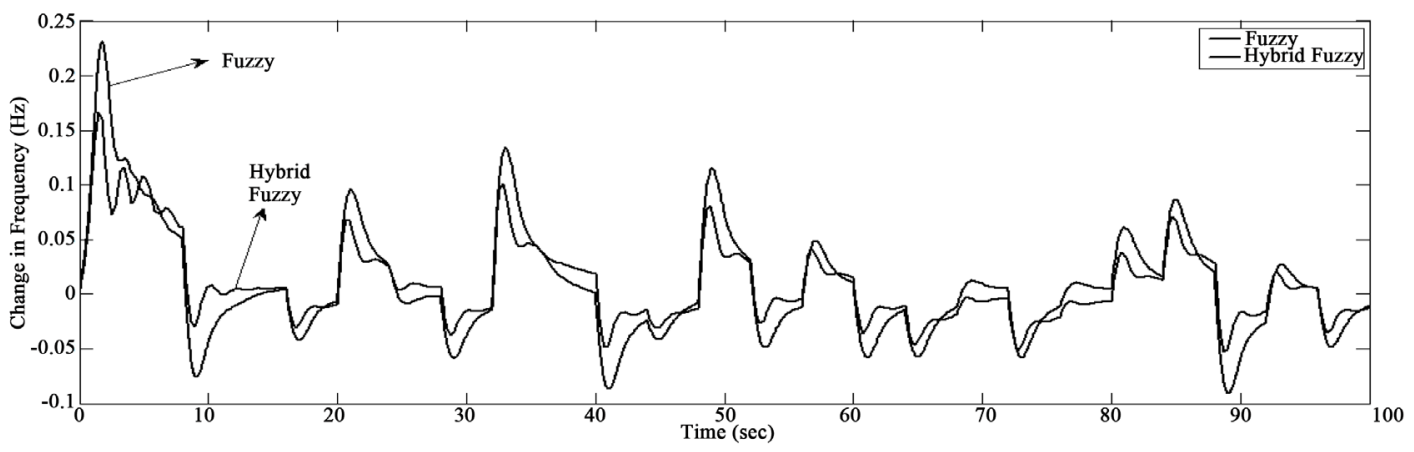

Figure 11. Frequency deviation in Area 1 (Case III).

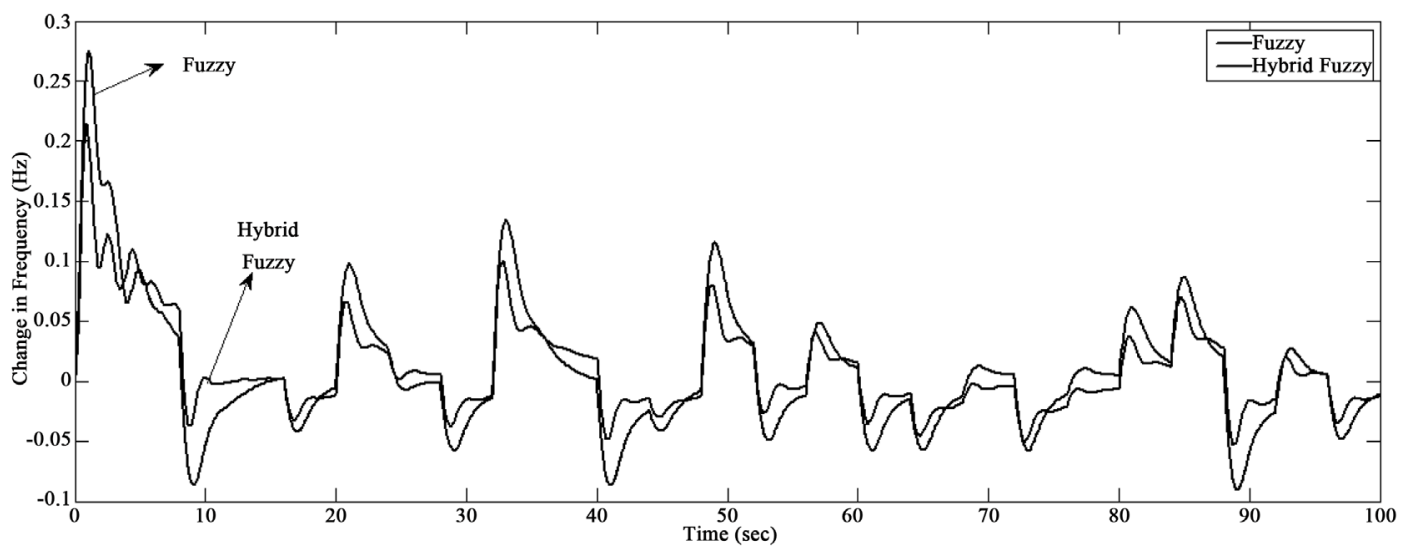

Figure 12. Frequency deviation in Area 2 (Case III).

Table 3. Gaussian MF parameters $\left(C_{n j}\right.$ and $\left.\sigma_{n j}\right)$ for Case 1.

\begin{tabular}{ccccc}
\hline \multirow{2}{*}{ Membership Functions } & \multicolumn{3}{c}{ Before PSO } & After PSO \\
\cline { 2 - 5 } & Mean $\left(C_{n j}\right)$ & Standard Deviation $\left(\sigma_{n j}\right)$ & Mean $\left(C_{n j}\right)$ & Standard Deviation $\left(\sigma_{n j}\right)$ \\
\hline NL & -0.667 & 0.118 & -1 & 0.07786 \\
NM & -0.3891 & 0.118 & -0.6833 & 0.04955 \\
NDS & -0.1114 & 0.118 & -0.3 & 0.04247 \\
Z & 0 & 0.118 & -0.05 & 0.04247 \\
PS & 0.444 & 0.118 & 0.3117 & 0.0304 \\
PM & 0.7221 & 0.118 & 0.6708 & 0.03575 \\
PL & 1 & 0.118 & 1 & 0.0354 \\
\hline
\end{tabular}


Table 4. Performance measures.

\begin{tabular}{|c|c|c|c|c|c|}
\hline \multirow{2}{*}{ Case } & \multirow{2}{*}{ Controller } & \multicolumn{2}{|c|}{ Area1 } & \multicolumn{2}{|c|}{ Area2 } \\
\hline & & Overshoot (Hz) & Settling Time (Sec) & Overshoot (Hz) & Settling Time (Sec) \\
\hline \multirow{2}{*}{1} & Fuzzy & -0.0785 & 42 & -0.08 & 28 \\
\hline & Hybrid Fuzzy & -0.048 & 25 & 0.05 & 20 \\
\hline \multirow{2}{*}{2} & Fuzzy & 0.13 & 48 & -0.13 & 42 \\
\hline & Hybrid Fuzzy & -0.042 & 28 & 0.07 & 26 \\
\hline \multirow{2}{*}{3} & Fuzzy & 0.17 & - & 0.27 & - \\
\hline & Hybrid Fuzzy & 0.1 & - & 0.2 & - \\
\hline
\end{tabular}

Table 5. Optimal value of controller parameter for Area 1 and Area 2.

\begin{tabular}{cccc}
\hline Case & Gain & Fuzzy & Hybrid Fuzzy \\
\hline & $K_{P 1}$ & 0.175 & 0.225 \\
$K_{I 1}$ & 0.250 & 0.285 \\
$K_{P 2}$ & 0.2331 & 0.203 \\
$K_{I 2}$ & 0.3701 & 0.485 \\
$K_{P 1}$ & 0.20 & 0.267 \\
& $K_{I 1}$ & 0.555 & 0.486 \\
& $K_{P 2}$ & 0.2051 & 0.165 \\
& $K_{I 2}$ & 0.685 & 0.455 \\
& $K_{P 1}$ & 0.210 & 0.206 \\
& $K_{I 1}$ & 0.545 & 0.525 \\
& $K_{P 2}$ & 0.2123 & 0.2 \\
& $K_{I 2}$ & 0.680 & 0.657 \\
\hline
\end{tabular}

\section{Conclusion}

In this paper, automatic tuning of membership function of fuzzy controller using PSO is applied to bilateral LFC scheme. PSO is a popular search algorithm; it utilizes the swarm intelligence of cooperation and fast convergence to find the optimum value of the parameters of membership function used in fuzzy logic controller. Simulation results show the effectiveness of the proposed controller in damping the frequency oscillations and tie-line power very fast with less undershoot and overshoot. It is also seen from simulation results that under normal circumstances, Fuzzy and Hybrid Fuzzy using PSO-based controllers produce the better results. During parameter uncertainties and large load disturbance, Hybrid Fuzzy penalizes excessively over damped and excessively under damped responses and reduces overshoots. It has been therefore shown that Hybrid Fuzzy controller produces better performance in all testing environments.

\section{References}

[1] Christie, R.D. and Bose, A. (1996) Load Frequency Control Issues in Power System Operations after Deregulation. IEEE Transactions on Power Systems, 11, 1191-1200. http://dx.doi.org/10.1109/59.535590

[2] Donde, V., Pai, M.A. and Hiskens, I.A. (2001) Simulation and Optimization in an AGC System after Deregulation. IEEE Transactions on Power Systems, 16, 481-489. http://dx.doi.org/10.1109/59.932285

[3] Bevrani, H., Mitani, Y., Tsuji, K. and Bevrani, H. (2005) Bilateral Based Robust Load Frequency Control. Energy 
Conversion and Management, 46, 1129-1146. http://dx.doi.org/10.1016/j.enconman.2004.06.024

[4] Delfino, B., Fornari, F. and Massucco, S. (2002) Load-Frequency Control and Inadvertent Interchange Evaluation in Restructured Power Systems. IEE Proceedings-Generation, Transmission and Distribution, 149, 607-614. http://dx.doi.org/10.1049/ip-gtd:20020368

[5] Bevrani, H., Mitani, Y. and Tsuji, K. (2004) Robust Decentralised AGC in Restructured Power System. Energy Conversion and Management, 45, 2297-2312,. http://dx.doi.org/10.1016/j.enconman.2003.11.018

[6] Shayeghi, H., Shayanfar, H.A. and Jalili, A. (2006) Multi Stage Fuzzy PID Power System Automatic Generation Controller in the Deregulated Environment. Energy Conversion and Management, 47, 2829-2845. http://dx.doi.org/10.1016/j.enconman.2006.03.031

[7] Shayeghi, H., Jalili, A. and Shayanfar, H.A. (2007) Robust Modified GA Based Multi-Stage Fuzzy LFC. Energy Conversion and Management, 48, 1656-1670. http://dx.doi.org/10.1016/j.enconman.2006.11.010

[8] Chaturvedi, D.K., Satsangi, P.S. and Kalra, P.K. (1999) Load Frequency Control: A Generalized Neural Network Approach. International Journal of Electrical Power \& Energy Systems, 21, 405-415. http://dx.doi.org/10.1016/S0142-0615(99)00010-1

[9] Nanda, J., Mishra, S. and Saikia, L.C. (2009) Maiden Application of Bacterial Foraging Based Optimization Technique in Multi-Area Automatic Generation Control. IEEE Transactions on Power Systems, 24, 602-609. http://dx.doi.org/10.1109/TPWRS.2009.2016588

[10] Bevrani, H. and Hiyama, T. (2011) Intelligent Automatic Generation Control. CRC Press, Boca Raton, 77-85.

[11] Daneshfar, F. (2013) Intelligent Load-Frequency Control in a Deregulated Environment: Continuous-Valued Input, Extended Classifier System Approach. IET Generation, Transmission \& Distribution, 7, 551-559. http://dx.doi.org/10.1049/iet-gtd.2012.0478

[12] Kundur, P. (2008) Power System Stability \& Control. 5th Reprint, Tata McGraw-Hill, New Delhi, 581-626.

[13] Saadat, H. (2010) Power System Analysis. Tata McGraw-Hill, New Delhi, 542-555.

[14] Ziegler, J.G. and Nichols, N.B. (1942) Optimum Settings for Automatic Controllers. ASME, 64, 59-768.

[15] Kennedy, J. and Eberhart, R. (1995) Particle Swarm Optimization. Proceedings of IEEE International Conference on Neural Networks, Vol. 4, 1942-1948. http://dx.doi.org/10.1109/icnn.1995.488968

[16] Clerc, M. and Kennedy, J. (2002) The Particle Swarm-Explosion, Stability, and Convergence in a Multidimensional Complex Space. IEEE Transactions on Evolutionary Computation, 6, 58-73. http://dx.doi.org/10.1109/4235.985692

[17] Kennedy, J., Eberhart, R. and Shi, Y. (2001) Swarm Intelligence. Morgan Kaufman Publishers, San Francisco.

\section{Appendix A}

$R_{1}=R_{3}=3 \% ; R_{2}=R_{4}=2.73 \% ; T_{g 1}=T_{g 3}=0.08 \mathrm{~s} ; T_{g 2}=T_{g 4}=0.06 \mathrm{~s} ; T_{t 1}=T_{t 3}=0.4 \mathrm{~s} ; T_{t 2}=T_{t 4}=0.44 \mathrm{~s} ;$ $K_{r 1}=K_{r 2}=K_{r 3}=K_{r 4}=0.5 ; T_{r 1}=T_{r 2}=T_{r 3}=T_{r 4}=10 \mathrm{~s} ; D_{1}=0.015 \mathrm{pu} \cdot \mathrm{MW} / \mathrm{Hz} ; D_{2}=0.016 \mathrm{pu} \cdot \mathrm{MW} / \mathrm{Hz} ; M_{1}=$ $0.16667 ; M_{2}=0.2012 ; B_{1}=0.3483 ; B_{2}=0.3827 ; T_{12}=0.20$. 